\title{
O'Leary-Sant Symptom Index Predicts the Treatment Outcome for Onabotulinumtoxin A Injections for Refractory Interstitial Cystitis/Bladder Pain Syndrome
}

\author{
Yuh-Chen Kuo ${ }^{1}$ and Hann-Chorng Kuo ${ }^{2, *}$
}

1 Department of Urology, Yangming Branch of Taipei City Hospital, 105 Yu-Sheng Street, Taipei 11148, Taiwan; E-Mail: yuhchens@hotmail.com

2 Department of Urology, Buddhist Tzu Chi General Hospital and Tzu Chi University, 707, Section 3, Chung Yang Road, Hualien 97002, Taiwan

* Author to whom correspondence should be addressed; E-Mail: hck@tzuchi.com.tw;

Tel.: +886-3-856-1825 (ext. 2117); Fax: +886-3-856-0794.

Academic Editor: Bahman Jabbari

Received: 15 June 2015 / Accepted: 27 July 2015 / Published: 30 July 2015

\begin{abstract}
Although intravesical injection of onabotulinumtoxinA (BoNT-A) has been proved promising in treating patients with interstitial cystitis/bladder pain syndrome (IC/BPS), what kind of patients that may benefit from this treatment remains unclear. This study investigated the predictors for a successful treatment outcome. Patients with IC/BPS who failed conventional treatments were enrolled to receive intravesical injection of $100 \mathrm{U}$ of BoNT-A immediately followed by hydrodistention. Variables such as O'Leary-Sant symptom and problem indexes (ICSI and ICPI), pain visual analogue scale (VAS), functional bladder capacity (FBC), voiding diary, and urodynamic parameters were measured at baseline and six months after treatment. A global response assessment (GRA) $\geq 2$ at six months was defined as successful. There were101 patients enrolled. Significant improvements were observed in mean ICSI, ICPI, OSS (ICSI + ICPI), pain VAS, FBC, frequency, nocturia and GRA at six months after BoNT-A injections (all $p<0.05$ ). The successful rate at six months was 46/101 (45.54\%). Multivariate logistic regression revealed the baseline ICSI (odds ratio $=0.770,95 \%$ confidence interval $=0.601-0.989$ ) was the only predictor for a treatment outcome. ICSI $\geq 12$ was the most predictive cutoff value for a treatment failure, with a ROC area of 0.70 (sensitivity $=69.1 \%$, specificity $=60.9 \%$ ).
\end{abstract}


Keywords: interstitial cystitis; onabotulinumtoxinA; treatment; predictive factors

\section{Introduction}

Interstitial cystitis/bladder pain syndrome (IC/BPS) is a debilitating chronic disease of unknown etiology characterized by urgency, frequency, and suprapubic discomfort during bladder filling. Current treatments are usually unsuccessful in completely eradicating bladder pain and increasing bladder capacity [1]. Intravesical resiniferatoxin once was considered to be effective but this has not been shown in a large scale multiple center trial [2]. Other intravesical therapies such as hyaluronic acid, bacilli Calmette-Guerine, and oral medications with pentosan polysulfate sodium, cyclosporine A, or amitriptyline have not been demonstrated to be long-term effective [3-6]. Until now, bladder hydrodistention is still the most popular treatment for IC/BPS refractory to conventional treatment. However, the effective duration of hydrodistention is usually short and repeated hydrodistention or conversion to other treatment such as botulinum toxin injection is necessary.

OnabotulinumtoxinA (BoNT-A) is one of the most powerful neurotoxins to inhibit the release of neurotransmitters from nerve fibers and urothelium [7,8]. Applications of BoNT-A for IC/BPS have been reported in some studies [9-13]. The results showed BoNT-A could reduce bladder pain, impair bladder sensation, and decrease chronic inflammation in the central nervous system in animal and human experiments. Although BoNT-A injection seems promising for treating symptoms of IC/BPS [14], most of the results are based on the data of mean changes of symptoms collected from the entire study cohort. There is still a certain percentage of patients who had unsuccessful outcomes after treatment. So far, which kind of patients will benefit from BoNT-A injection remains unclear.

Identification of the predictive factors for a successful treatment outcome may help differentiating the subset of IC/BPS patients who will not respond to single BoNT-A injection. We could then avoid giving these patients ineffective procedures and exposing them to potential treatment-related adverse effects. Additionally, we might further counsel and encourage them to receive more advanced treatment options such as repeated BoNT-A injections or enterocystoplasty. We conducted this study to evaluate if there are clinical variables that can predict a treatment success after a single injection of BoNT-A for the treatment of IC/BPS refractory to conventional treatment.

\section{Results and Discussion}

From October 2005 to October 2013, 101 patients with diagnosis of IC/BPS who have failed conventional treatments were enrolled in this study. Among them, there were 13 men and 88 women who aged $46.00 \pm 13.27$ (27-72) and $48.81 \pm 11.81$ (21-78) years, respectively. The demographics of these subjects are described in Table 1. There was no significant difference between either sex in distribution of age, IC symptom scores, pain visual analogue scale (VAS), functional bladder capacity (FBC), daytime frequency, nocturia, urodynamic parameters, result of potassium sensitivity test (PST) and cystoscopic parameters. 
Table 1. Demographics of the patients.

\begin{tabular}{ccccc}
\hline Parameters & Total $(\boldsymbol{n}=\mathbf{1 0 1})$ & Male $(\boldsymbol{n}=\mathbf{1 3})$ & Female $(\boldsymbol{n}=\mathbf{8 8})$ & $\boldsymbol{p}$ Value \\
\hline Age (years) & $48.45 \pm 11.97$ & $46.00 \pm 13.27$ & $48.81 \pm 11.81$ & 0.433 \\
ICSI & $12.34 \pm 3.42$ & $11.08 \pm 3.95$ & $12.52 \pm 3.32$ & 0.156 \\
ICPI & $11.43 \pm 3.03$ & $11.77 \pm 3.19$ & $11.38 \pm 3.02$ & 0.664 \\
OSS (ICSI + ICPI) & $23.76 \pm 6.14$ & $22.85 \pm 6.59$ & $23.90 \pm 6.09$ & 0.567 \\
Pain VAS & $5.23 \pm 2.37$ & $4.23 \pm 3.24$ & $5.38 \pm 2.20$ & 0.105 \\
FBC & $128.86 \pm 75.37$ & $156.92 \pm 58.93$ & $124.72 \pm 76.91$ & 0.151 \\
Frequency & $15.39 \pm 7.68$ & $13.69 \pm 6.10$ & $15.64 \pm 7.88$ & 0.397 \\
Nocturia & $4.70 \pm 4.67$ & $3.38 \pm 1.85$ & $4.90 \pm 4.93$ & 0.278 \\
FSF & $115.96 \pm 53.40$ & $105.92 \pm 55.63$ & $117.33 \pm 53.28$ & 0.490 \\
FD & $171.19 \pm 73.44$ & $192.20 \pm 72.48$ & $168.53 \pm 73.59$ & 0.340 \\
SD & $202.46 \pm 87.01$ & $233.67 \pm 79.81$ & $197.45 \pm 87.74$ & 0.249 \\
CBC & $273.98 \pm 109.32$ & $268.83 \pm 109.71$ & $274.72 \pm 109.92$ & 0.863 \\
Pdet & $19.87 \pm 10.82$ & $24.33 \pm 11.39$ & $19.19 \pm 10.65$ & 0.126 \\
Qmax & $12.56 \pm 5.38$ & $12.17 \pm 4.86$ & $12.62 \pm 5.48$ & 0.787 \\
Volume & $241.18 \pm 110.54$ & $219.67 \pm 101.13$ & $244.41 \pm 112.12$ & 0.473 \\
PVR & $39.38 \pm 94.08$ & $49.17 \pm 103.61$ & $38.01 \pm 93.26$ & 0.703 \\
Positive PST & $98(97.03)$ & $12(92.31)$ & $86(97.73)$ & $0.342{ }^{1}$ \\
Cystoscopic HD & & & & \\
MBC (mL) & $570.00 \pm 198.07$ & $603.85 \pm 145.00$ & $681.82 \pm 218.30$ & 0.216 \\
Hunner's ulcer & $10(9.90)$ & $0(0)$ & $10(11.36)$ & $0.200{ }^{1}$ \\
Glomerulation & & & & $0.386{ }^{1}$ \\
Grade 0 & $8(7.92)$ & $1(7.69)$ & $7(7.95)$ & \\
Grade 1 & $39(38.61)$ & $7(53.85)$ & $32(36.36)$ & \\
Grade 2 & $35(34.65)$ & $3(23.08)$ & $32(36.36)$ & \\
Grade 3 & $11(10.89)$ & $0(0)$ & $11(12.50)$ & \\
Grade 4 & $8(7.92)$ & $2(15.38)$ & $6(6.82)$ & \\
\hline
\end{tabular}

Note: ${ }^{1}$ Chi-square test. Otherwise, the independent $t$ test was used; ICSI: O'Leary-Sant IC Symptom Index; ICPI: IC Problem Index; VAS: Visual analogue scale. FBC: Functional bladder capacity; FSF: First sensation of filling; FD: First desire to void. SD: Strong desire to void; CBC: Cystometric bladder capacity; Pdet: Detrusor pressure at Qmax; Qmax: Maximum flow rate; PVR: Postvoid residual; PST: Potassium sensitivity test; HD: Hydrodistention. MBC: Maximal bladder capacity.

Table 2 listed the changes of clinical parameters at baseline, three and six months after a single injection of BoNT-A. For all of the patients, significant improvements could be observed in mean O'Leary-Sant IC Symptom Index (ICSI) and IC Problem Index (ICPI), OSS (ICSI + ICPI), pain VAS, FBC, frequency, nocturia, and global response assessment (GRA) at three months after BoNT-A injections. These improvements continued to exist at six months which demonstrated the durable efficacy of BoNT-A injection on the treatment of refractory IC/BPS.

At six months after BoNT-A injection, the overall therapeutic results were reported to be failed in $55(54.46 \%)$ and successful in $46(45.54 \%)$ patients by GRA criteria (GRA $\geq 2$ as successful) (Table 3). Univariate logistic regression analysis revealed significant relationships between the treatment outcome and baseline ICSI, ICPI, OSS, FBC, frequency, and the urodynamic parameter, first desire to void (FD) (all $p<0.05$ ). That is, the subjects with lower ICSI, ICPI, OSS scores, lower frequency times, higher 
mean amount of FBC or higher mean volume of FD at baseline had a significantly greater chance to achieve a successful treatment outcome. In addition, the multivariate logistic regression model was performed based on all significant variables in the univariate analysis and showed that the baseline ICSI score (odds ratio $=0.770,95 \%$ confidence interval $=0.601-0.989$ ) was the only predictive factor for a treatment outcome in refractory IC/BPS patients (Table 4). We found ICSI $\geq 12$ was the most predictive cutoff value for treatment failure, with a ROC area of 0.70 (sensitivity $=69.1 \%$, specificity $=60.9 \%$ ) (Figure 1).

Table 2. The changes of Parameters at baseline, 3 and 6 months after single BoNT-A injection.

\begin{tabular}{ccccc}
\hline Parameters $(\boldsymbol{N = 1 0 1})$ & Baseline & 3 Months & 6 Months & $\boldsymbol{p}$ Value $^{\mathbf{1}}$ \\
\hline ICSI & $12.34 \pm 3.42$ & $8.92 \pm 4.42$ & $8.79 \pm 4.65$ & 0.000 \\
ICPI & $11.43 \pm 3.03$ & $7.51 \pm 4.30$ & $7.75 \pm 4.57$ & 0.000 \\
OSS (ICSI+ICPI) & $23.76 \pm 6.14$ & $16.43 \pm 8.51$ & $16.54 \pm 8.95$ & 0.000 \\
Pain VAS & $5.23 \pm 2.37$ & $3.45 \pm 2.47$ & $3.50 \pm 2.54$ & 0.000 \\
FBC & $128.86 \pm 75.37$ & $180.00 \pm 90.53$ & $177.92 \pm 86.39$ & 0.000 \\
Frequency & $15.39 \pm 7.68$ & $11.79 \pm 6.35$ & $11.28 \pm 6.29$ & 0.000 \\
Nocturia & $4.70 \pm 4.67$ & $3.31 \pm 2.86$ & $3.52 \pm 3.79$ & 0.026 \\
Qmax & $12.56 \pm 5.38$ & $13.97 \pm 6.07$ & $14.53 \pm 5.91$ & 0.060 \\
Volume & $241.18 \pm 110.54$ & $236.66 \pm 123.27$ & $236.50 \pm 121.70$ & 0.955 \\
PVR & $39.38 \pm 94.08$ & $48.03 \pm 79.09$ & $48.09 \pm 77.48$ & 0.711 \\
CBC & $273.98 \pm 109.32$ & $285.64 \pm 140.59$ & $284.59 \pm 142.12$ & 0.799 \\
GRA & 0 & $1.27 \pm 1.00$ & $1.32 \pm 0.97$ & 0.000 \\
\hline
\end{tabular}

Note: ${ }^{1}$ Wilcoxon rank-sum test; ICSI: O’Leary-Sant IC Symptom Index; ICPI: IC Problem Index; VAS: Visual analogue scale; FBC: Functional bladder capacity; Qmax: Maximum flow rate; PVR: Postvoid residual; CBC: Cystometric bladder capacity; GRA: Global response assessment.

Table 3. Univariate logistic regression of parameters associated with the treatment outcome of intravesical BoNT-A injection for IC/BPS.

\begin{tabular}{ccccc}
\hline \multirow{2}{*}{ Parameters } & \multicolumn{2}{c}{ Treatment Outcome } & \multirow{2}{*}{ OR (95\% CI) } & \multirow{2}{*}{$\boldsymbol{p}$ Value } \\
\cline { 2 - 3 } & Successful & Failed & & \\
No. patients & 46 & 55 & & \\
Gender & & & & \\
Male (\%) & $5(38.46)$ & $8(61.54)$ & $1.396(0.423-4.603)$ & 0.584 \\
Female (\%) & $41(46.59)$ & $47(53.41)$ & & \\
Age (years) & $46.91 \pm 12.00$ & $49.73 \pm 11.91$ & $0.980(0.948-1.014)$ & 0.240 \\
ICSI & $11.15 \pm 3.47$ & $13.33 \pm 3.07$ & $0.811(0.709-0.928)$ & 0.002 \\
ICPI & $10.76 \pm 3.34$ & $11.98 \pm 2.65$ & $0.869(0.756-0.999)$ & 0.048 \\
OSS (ICSI+ICPI) & $21.91 \pm 6.56$ & $25.31 \pm 5.35$ & $0.905(0.756-0.999)$ & 0.008 \\
Pain VAS & $4.80 \pm 2.25$ & $5.58 \pm 2.43$ & $0.866(0.729-1.030)$ & 0.104 \\
FBC & $151.96 \pm 82.75$ & $109.55 \pm 63.09$ & $1.008(1.002-1.014)$ & 0.007 \\
Frequency & $13.37 \pm 5.02$ & $17.07 \pm 9.04$ & $0.922(0.861-0.989)$ & 0.023 \\
Nocturia & $3.59 \pm 2.54$ & $5.64 \pm 5.75$ & $0.852(0.724-1.002)$ & 0.052 \\
VUDS parameters & & & & \\
FSF (mL) & $123.93 \pm 49.57$ & $109.17 \pm 56.03$ & $1.005(0.998-1.013)$ & 0.169 \\
FD (mL) & $191.12 \pm 68.71$ & $154.17 \pm 73.74$ & $1.007(1.001-1.014)$ & 0.021 \\
\hline
\end{tabular}


Table 3. Cont.

\begin{tabular}{|c|c|c|c|c|}
\hline \multirow{2}{*}{ Parameters } & \multicolumn{2}{|c|}{ Treatment Outcome } & \multirow{2}{*}{ OR $(95 \%$ CI) } & \multirow{2}{*}{$p$ Value } \\
\hline & Successful & Failed & & \\
\hline $\mathrm{SD}(\mathrm{mL})$ & $222.64 \pm 83.10$ & $181.66 \pm 87.31$ & $1.006(1.000-1.012)$ & 0.062 \\
\hline $\mathrm{CBC}(\mathrm{mL})$ & $289.50 \pm 107.99$ & $259.41 \pm 109.66$ & $1.003(0.999-1.006)$ & 0.181 \\
\hline $\operatorname{Pdet}\left(\mathrm{cm} \mathrm{H}_{2} \mathrm{O}\right)$ & $19.51 \pm 10.39$ & $20.19 \pm 11.30$ & $0.994(0.957-1.033)$ & 0.765 \\
\hline $\mathrm{Qmax}(\mathrm{mL} / \mathrm{s})$ & $13.03 \pm 4.81$ & $12.11 \pm 5.89$ & $1.033(0.955-1.117)$ & 0.416 \\
\hline Voided volume (mL) & $264.82 \pm 110.28$ & $219.52 \pm 107.38$ & $1.004(1.000-1.008)$ & 0.052 \\
\hline PVR (mL) & $32.60 \pm 91.79$ & $42.19 \pm 96.87$ & $0.999(0.995-1.004)$ & 0.752 \\
\hline \multicolumn{5}{|l|}{ PST } \\
\hline Negative (\%) & $1(33.33)$ & $2(66.67)$ & & \\
\hline Positive (\%) & 45 (45.92) & $53(54.08)$ & $1.698(0.149-19.349)$ & 0.670 \\
\hline \multicolumn{5}{|l|}{ Cystoscopic HD } \\
\hline $\mathrm{MBC}(\mathrm{mL})$ & $688.04 \pm 199.77$ & $658.18 \pm 221.48$ & $1.001(0.999-1.003)$ & 0.478 \\
\hline \multicolumn{5}{|l|}{ Hunner's ulcer } \\
\hline Negative (\%) & $43(47.25)$ & $48(52.75)$ & & \\
\hline Positive (\%) & $3(30.00)$ & $7(70.00)$ & $0.307(0.756-0.999)$ & 0.307 \\
\hline \multicolumn{5}{|l|}{ Glomerulation } \\
\hline Grade $0(\%)$ & $6(75.00)$ & $2(25.00)$ & & \\
\hline Grade $1(\%)$ & $15(38.46)$ & $24(61.54)$ & $0.208(0.037-1.170)$ & 0.075 \\
\hline Grade $2(\%)$ & $18(51.43)$ & $17(48.57)$ & $0.353(0.062-1.995)$ & 0.239 \\
\hline Grade $3(\%)$ & $4(36.36)$ & $7(63.64)$ & $0.190(0.025-1.432)$ & 0.107 \\
\hline Grade $4(\%)$ & $3(37.50)$ & $5(62.50)$ & $0.200(0.023-1.712)$ & 0.142 \\
\hline
\end{tabular}

Note: OR: Odds ratio; CI: Confidence interval; ICSI: O'Leary-Sant IC Symptom Index; ICPI: IC Problem Index; VAS: Visual analogue scale; FBC: Functional bladder capacity; FSF: First sensation of filling; FD: First desire to void; SD: Strong desire to void; CBC: Cystometric bladder capacity; Pdet: Detrusor pressure at Qmax; Qmax: Maximum flow rate; PVR: Postvoid residual; PST: Potassium sensitivity test; HD: Hydrodistention; MBC: Maximal bladder capacity.

Table 4. Multivariate analysis of parameters significantly associated with a successful treatment outcome on univariate analysis.

\begin{tabular}{cccc}
\hline Parameters & Odds Ratio & $\mathbf{9 5 \%}$ CI & $\boldsymbol{p}$ Value \\
\hline ICSI & 0.770 & $0.601-0.989$ & 0.040 \\
ICPI & 1.106 & $0.853-1.435$ & 0.447 \\
FBC $(\mathrm{mL})$ & 1.003 & $0.996-1.010$ & 0.389 \\
Frequency & 1.011 & $0.904-1.053$ & 0.531 \\
FD $(\mathrm{mL})$ & 1.005 & $0.998-1.012$ & 0.171 \\
\hline
\end{tabular}

Note: CI: Confidence interval; ICSI: O'Leary-Sant IC Symptom Index; ICPI: IC Problem Index; FBC: Functional bladder capacity; FD: First desire to void.

So far the pathogenesis of IC/BPS is not fully understood. The management of IC/BPS is directed mainly to amelioration of the bothersome symptoms such as bladder pain or urinary tract symptoms. This study demonstrated that intravesical BoNT-A injection could significantly relieve the bothersome symptoms (ICSI, ICPI, OSS, pain VAS, daytime frequency and nocturia) and increase the functional 
bladder capacity in patients with refractory IC/BPS at 3 and 6 months after treatment. These effects are comparable to previous reports [11-13,15].

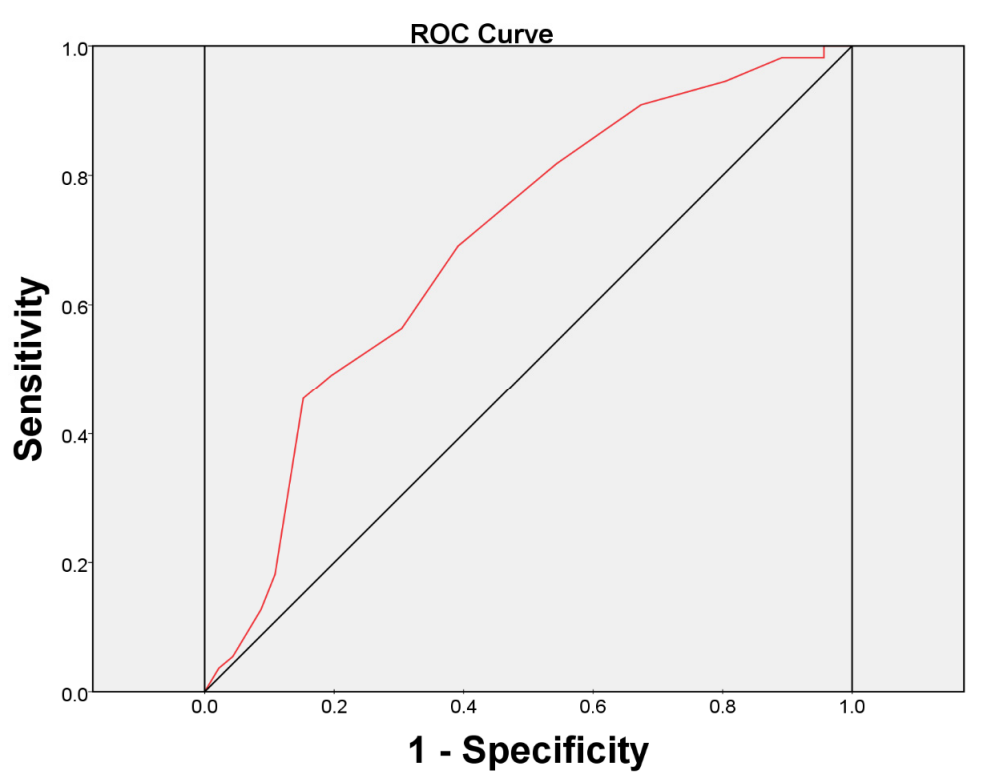

Figure 1. The area under the receiver operating characteristic (ROC) curve for baseline ICSI score $\geq 12$ as a prognostic test for the patients with a failed treatment outcome.

BoNT-A acts by cleaving the SNAP-25 (Synaptosome-associated protein of $25 \mathrm{kd}$ ) complex in the presynaptic terminal, which prevents formation of the SNARE system. By this mechanism, the neurotransmitter vesicles cannot work at the presynaptic membrane, which decreases the release of neurotransmitters at the synaptic cleft. Consequently, the release of acetylcholine, calcitonin gene-related protein, substance-P, and glutamate decreased and the nociceptive fiber discharge reduced [16,17], suggesting the therapeutic rationale of BoNT-A on IC/BPS. Furthermore, recent studies have demonstrated that BoNT-A has an anti-inflammatory effect on a cystitis rat model [18] and that injection of BoNT-A can reduce the production of nerve growth factor in bladder resulting in satisfactory pain relief in IC/PBS patients $[19,20]$.

Pilot studies of intravesical injection of BoNT-A for IC/BPS had been introduced since 2004 with variable successful rates. Smith et al. reported a $69 \%$ success rate with a therapeutic duration of nine months [9]. Giannantoni et al. reported $85.7 \%$ of patients had an improvement at three months [11]. Our previous study revealed that single intravesical injections of BoNT-A, followed by hydrodistention, produced significantly better clinical results than hydrodistention alone [21]. Of the patients $71 \%$ had a successful result at six months. In the current study, the overall success rate of treatment at six months was $45.54 \%$ according to GRA $\geq 2$. The large variation in successful rates between the above studies may reflect the differences of the study population, disease severity, definition of success, dosage of BoNT-A, injection method, and so on, in different study cohorts. However, these reports are similar in one fact: not all the IC/BPS patients benefit from BoNT-A injection. This result implies the need for further research on the causes of a treatment failure or the ways to differentiate which IC/BPS patient will take the advantage of BoNT-A injection. 
Based on our findings from univariate logistic regression analysis, the patients who failed BoNT-A injection at six months possessed higher baseline ICSI, ICPI, OSS, frequency, and lower mean volume of FBC and FD than those who succeeded in treatment. However, using multivariate logistic regression model, baseline ICSI was found to be the only independent predictive factor for a successful treatment of BoNT-A injection. The reason for this is not clear. It is possible that a higher symptom score (ICSI $\geq 12$ ) may reflect more advanced urothelial dysfunction and neurogenic inflammation of the bladder leading to more unpleasant sensation during bladder filling, and possibly more severe fibrosis of the bladder wall which could not be resolved completely by single injection of BoNT-A. Since the components of ICSI questionnaire consist of four domains, urgency, frequency, nocturia, and pain, our finding suggested that the overall patients' perception, ICSI, but not individual symptom or clinical variable before treatment will predict the treatment outcome of BoNT-A injection for refractory IC/BPS. To our knowledge, this is the first study to discover the prognostic factor of intravesical BoNT-A injection for the treatment of IC/BPS.

The results of this study also point out that only $45.54 \%$ of patients have a successful treatment from a single BoNT-A injection. Although BoNT-A has anti-inflammation and anti-nociceptive effects, a single injection might not completely resolve the chronic inflammation residing in the bladder wall. This result is also reflected by the previous studies that the therapeutic effect is fading with time and no patient can have effect at 12 months after 200 U BoNT-A single injection [12], and repeated BoNT-A injections provides a significantly better long-term effect compared with a single injection $[22,23]$.

Recently, Denys et al. observed that neurogenic detrusor overactivity (NDO) patients without response at a first onabotulinumtoxin injection had a good response at a second injection [24]. Also, NDO patients who failed after abobotulinum toxin in 20 sites had good responses after an equivalent onabotulinum toxin injection in 30 sites [25]. The quality of injections and reconstitution of onabotulinum toxin may have an influence on the effect of the first BoNT-A injection. As such, one may argue that we can assume failure after a single injection. In our study, all the injections were prepared and performed by a single operator (Kuo, H.C.) with more than ten years' experience in BoNT-A injection. The treatment procedure has been standardized and risk of inappropriate injection has been minimized so that the treatment outcome can be compared on this basis.

During bladder inflammation, the exocytosis of TRPV1 increases and plays a role in the perception of thermal and inflammatory pain [26]. BoNT-A could block the TRPV1 trafficking to the membrane during bladder inflammation and inhibit the inflammatory sensitization of TRPV1 [27]. Intravesical onabotulinumtoxinA administration could inhibit the evoked ATP release from urothelium of chronic bladder inflammation models [28]. BoNT-A administration to the rat bladder can also decrease the amount of spinal cord C-fos expression due to chronic bladder inflammation [29]. One proposed mechanism of BoNT-A induced analgesia is the combination of direct analgesic effect by its action on peripheral nociceptive neurons and indirect analgesic affect by the retrograde transport of toxin to prevent central sensitization in patients with chronic pain [30]. A recent randomized, double-blind, placebo-controlled, multicentre trial showed that bladder injections of $100 \mathrm{U}$ of BoNT-A effectively reduced bladder pain symptoms in patients with IC/BPS. At week eight, a significantly greater reduction in pain score was observed in the BoNT-A group compared to the normal-saline group $(-2.6 \pm 2.8$ vs. $-0.9 \pm 2.2, p=0.021)$. Adverse events did not differ between the groups [31]. 
The major limitations of our present study included the lack of a control arm and that it was not randomized. Although an ICSI score of 12 was found to be the best cutoff value for predicting therapeutic efficacy, the clinical implication of this cutoff value is limited by its small area under the ROC curve, and its low sensitivity and specificity. We should realize that even in patients with high ICSI, some may have a good treatment response. Nevertheless, these findings could serve as an initial guide or assist in consultation regarding the treatment of IC/BPS patients with BoNT-A. Further well-designed randomized trials to confirm the results of this study are needed.

\section{Experimental Section}

\subsection{Patient Enrollment}

Patients with IC/BPS who have failed conventional treatments have been enrolled in this study. They have been treated with oral pentosan polysulfate sodium, intravesical instillation of heparin, hyaluronic acid, or oral tricyclic antidepressant for at least six months, but the symptoms remained unchanged or relapsed. A diagnosis of IC/BPS has been established based on characteristic symptoms and cystoscopic findings of glomerulation, petechia, or mucosal ulceration [32].

\subsection{Outcome Assessment}

Patients were requested to complete a three-day voiding diary before and after treatment. The IC/BPS symptoms were assessed by the O'Leary-Sant IC Symptom Index (ICSI) and IC Problem Index (ICPI) [33]. The pain score was reported by patient self-assessment using a 10-point visual analogue scale (VAS) system. Video-urodynamic study (Video UDS) and PST were performed. The treatment outcome was assessed using the GRA [34]. Patients were requested to rate symptoms compared with baseline on a seven-point centered scale from markedly, moderately and slightly worse, no change, to slightly, moderately and markedly improved. Patients with moderately and markedly improved results after treatment $(\mathrm{GRA} \geq 2)$ were considered to have a successful treatment outcome. Otherwise, the treatment was considered to have failed.

\subsection{Urodynamic Study}

UDS was performed by standard procedures using a 6 Fr dual channel catheter and an 8 Fr rectal balloon catheter. Cystometric study was performed with normal saline at a filling rate of $20 \mathrm{~mL} / \mathrm{min}$. All descriptions and terminology in this report were in accordance with the recommendations of the International Continence Society [35]. The urodynamic parameters included first sensation of bladder filling (FSF), first desire to void (FD), strong desire to void (SD), cystometric bladder capacity (CBC), maximum flow rate (Qmax), detrusor pressure at Qmax (Pdet) and postvoid residual (PVR). After the VUDS, $40 \mathrm{~mL} \mathrm{KCl}$ solution of $0.4 \mathrm{M}$ was infused slowly into the bladder and the test was regarded as positive when painful (of $\geq 2$ VAS score) or urgency sensation was elicited compared to normal saline infusion during urodynamic study. 


\subsection{Botulinum Toxin Injections}

The patients were admitted for the treatment. They received intravesical injection of $100 \mathrm{U}$ of BoNT-A (BOTOX, Allergan, Irvine, CA, USA) immediately followed by cystoscopic hydrodistention under intravenous general anesthesia. Each vial of BoNT-A was diluted with $10 \mathrm{ml}$ of normal saline, resulting in $10 \mathrm{U}$ BoNT-A per $1.0 \mathrm{~mL}$. Patients received 20 sites of suburothelial injection, which contained $5 \mathrm{U}$ in $0.5 \mathrm{~mL}$ for each site. The injection needle was inserted about $1 \mathrm{~mm}$ into the urothelium at the posterior and lateral walls of the bladder, using a 23 gauge needle and rigid cystoscopic injection instrument (22 Fr, Richard Wolf, Knittlingen, Germany). Cystoscopic hydrodistention was performed to an intravesical pressure of $80 \mathrm{~cm}$ water for $15 \mathrm{~min}$ immediately after the injection and the maximal bladder capacity (MBC) under hydrodistention was recorded.

\subsection{Patient Follow-up}

The patients were followed up every three months after treatment. The score of ICSI and ICPI, information on functional bladder capacity (FBC), daily urinary frequency, nocturia, pain VAS, and UDS parameters were recorded. At six months after the initial BoNT-A injection the patients were questioned of bladder conditions.

\subsection{Statistical Analysis}

The results of the voiding diary, UDS, ICSI, ICPI, and pain VAS were compared between baseline and each follow-up time point. Statistical comparisons between the groups were tested using a chi-square test for categorical variables, and an independent $t$ test or a Wilcoxon rank-sum test for continuous variables. Univariate and multivariate logistic regression analyses were used to identify variables predicting treatment success at six months after BoNT-A injection. The most predictive cutoff value of each variable was determined by the receiver operating characteristic curve (ROC) analysis. Statistical assessments were considered significant when $p<0.05$. Statistical analyses were performed using SPSS 18.0 statistical software (SPSS Inc., Chicago, IL, USA).

\section{Conclusions}

Baseline ICSI score represents the independent predictor for treatment success after BoNT-A injection. Patients with an ICSI of 12 or greater may have a poorer treatment outcome.

\section{Author Contributions}

Y.-C.K. analyzed data and was the primary author of the manuscript. H.-C.K. designed, oversaw, and performed the experiment and supervised the manuscript.

\section{Conflicts of Interest}

The authors declare no conflict of interest. 


\section{References}

1. Hanno, P.M.; Sant, G.R. Clinical highlights of the national institute of diabetes and digestive and kidney diseases/interstitial cystitis association scientific conference on interstitial cystitis. Urology 2001, 57, 2-6.

2. Payne, C.K.; Mosbaugh, P.G.; Forrest, J.B.; Evans, R.J.; Whitmore, K.E.; Antoci, J.P.; Perez-Marrero, R.; Jacoby, K.; Diokno, A.C.; O’Reilly, K.J.; et al. Intravesical resiniferatoxin for the treatment of interstitial cystitis: A randomized, double-blind, placebo controlled trial. J. Urol. 2005, 173, 1590-1594.

3. Nickel, J.C.; Barkin, J.; Forrest, J.; Mosbaugh, P.G.; Hernandez-Graulau, J.; Kaufman, D.; Lloyd, K.; Evans, R.J.; Parsons, C.L.; Atkinson, L.E. Randomized, double-blind, dose-ranging study of pentosan polysulfate sodium for interstitial cystitis. Urology 2005, 65, 654-658.

4. Sant, G.R.; Propert, K.J.; Hanno, P.M.; Burks, D.; Culkin, D.; Diokno, A.C.; Hardy, C.; Landis, J.R.; Mayer, R.; Madigan, R.; et al. A pilot clinical trial of oral pentosan polysulfate and oral hydroxyzine in patients with interstitial cystitis. J. Urol. 2003, 170, 810-815.

5. Hanno, P.M.; Buehler, J.; Wein, A.J. Use of amitriptyline in the treatment of interstitial cystitis. J. Urol. 1989, 141, 846-848.

6. Sairanen, J.; Forsell, T.; Ruutu, M. Long-term outcome of patients with interstitial cystitis treated with low dose cyclosporine A. J. Urol. 2004, 171, 2138-2141.

7. Rapp, D.E.; Turk, K.W.; Bales, G.T.; Cook, S.P. Botulinum toxin type a inhibits calcitonin gene-related peptide release from isolated rat bladder. J. Urol. 2006, 175, 1138-1142.

8. Chuang, Y.C.; Yoshimura, N.; Huang, C.C.; Chiang, P.H.; Chancellor, M.B. Intravesical botulinum toxin a administration produces analgesia against acetic acid induced bladder pain responses in rats. J. Urol. 2004, 172, 1529-1532.

9. Smith, C.P.; Radziszewski, P.; Borkowski, A.; Somogyi, G.T.; Boone, T.B.; Chancellor, M.B. Botulinum toxin a has antinociceptive effects in treating interstitial cystitis. Urology 2004, 64, 871-875.

10. Kuo, H.C. Preliminary results of suburothelial injection of botulinum a toxin in the treatment of chronic interstitial cystitis. Urol. Int. 2005, 75, 170-174.

11. Giannantoni, A.; Costantini, E.; Di Stasi, S.M.; Tascini, M.C.; Bini, V.; Porena, M. Botulinum a toxin intravesical injections in the treatment of painful bladder syndrome: A pilot study. Eur. Urol. 2006, 49, 704-709.

12. Giannantoni, A.; Porena, M.; Costantini, E.; Zucchi, A.; Mearini, L.; Mearini, E. Botulinum a toxin intravesical injection in patients with painful bladder syndrome: 1-year followup. J. Urol. 2008, 179, 1031-1034.

13. Chung, S.D.; Kuo, Y.C.; Kuo, H.C. Intravesical onabotulinumtoxinA injections for refractory painful bladder syndrome. Pain Phys. 2012, 15, 197-202.

14. Chrysanthopoulou, E.L.; Doumouchtsis, S.K. Challenges and current evidence on the management of bladder pain syndrome. Neurourol. Urodyn. 2014, 33, 1193-1201.

15. Pinto, R.; Lopes, T.; Frias, B.; Silva, A.; Silva, J.A.; Silva, C.M.; Cruz, C.; Cruz, F.; Dinis, P. Trigonal injection of botulinum toxin a in patients with refractory bladder pain syndrome/interstitial cystitis. Eur. Urol. 2010, 58, 360-365. 
16. Duggan, M.J.; Quinn, C.P.; Chaddock, J.A.; Purkiss, J.R.; Alexander, F.C.; Doward, S.; Fooks, S.J.; Friis, L.M.; Hall, Y.H.; Kirby, E.R.; et al. Inhibition of release of neurotransmitters from rat dorsal root ganglia by a novel conjugate of a clostridium botulinum toxin a endopeptidase fragment and erythrina cristagalli lectin. J. Biol. Chem. 2002, 277, 34846-34852.

17. Meng, J.; Wang, J.; Lawrence, G.; Dolly, J.O. Synaptobrevin I mediates exocytosis of CGRP from sensory neurons and inhibition by botulinum toxins reflects their anti-nociceptive potential. J. Cell Sci. 2007, 120, 2864-2874.

18. Lucioni, A.; Bales, G.T.; Lotan, T.L.; McGehee, D.S.; Cook, S.P.; Rapp, D.E. Botulinum toxin type a inhibits sensory neuropeptide release in rat bladder models of acute injury and chronic inflammation. BJU Int. 2008, 101, 366-370.

19. Liu, H.T.; Kuo, H.C. Intravesical botulinum toxin a injections plus hydrodistension can reduce nerve growth factor production and control bladder pain in interstitial cystitis. Urology 2007, 70, 463-468.

20. Liu, H.T.; Tyagi, P.; Chancellor, M.B.; Kuo, H.C. Urinary nerve growth factor level is increased in patients with interstitial cystitis/bladder pain syndrome and decreased in responders to treatment. BJU Int. 2009, 104, 1476-1481.

21. Kuo, H.C.; Chancellor, M.B. Comparison of intravesical botulinum toxin type a injections plus hydrodistention with hydrodistention alone for the treatment of refractory interstitial cystitis/painful bladder syndrome. BJU Int. 2009, 104, 657-661.

22. Kuo, H.C. Repeated intravesical onabotulinumtoxinA injections are effective in treatment of refractory interstitial cystitis/bladder pain syndrome. Int. J. Clin. Pract. 2013, 67, 427-434.

23. Kuo, H.C. Repeated onabotulinumtoxinA injections provide better results than single injection in treatment of painful bladder syndrome. Pain Phys. 2013, 16, E15-E23.

24. Denys, P.; Dmochowski, R.; Aliotta, P.; Castro-Diaz, D.; Blok, B.; Ethans, K.; Joshi, M.; Ni, Q.; Kennelly, M.Positive response to first onabotulinumtoxinA treatment persists long-term with repeattreatments in patients with neurogenic detrusor overactivity. Eur. Urol. Suppl. 2015, 14, e1092.

25. Peyronnet, B.; Roumiguié, M.; Castel-Lacanal, E.; Guillotreau, J.; Malavaud, B.; Marque, P.; Rischmann, P.; Gamé, X. Preliminary results of botulinum toxin A switch after first detrusor injection failure as a treatment of neurogenic detrusor overactivity. Neurourol. Urodyn. 2014, doi:10.1002/nau.22712.

26. Camprubi-Robles, M.; Planells-Cases, R.; Ferrer-Montiel, A. Differential contribution of SNARE-dependent exocytosis to inflammatory potentiation of TRPV1 in nociceptors. FASEB J. 2009, 23, 3722-3733.

27. Morenilla-Palao, C.; Planells-Cases, R.; Garcia-Sanz, N.; Ferrer-Montiel, A. Regulated exocytosis contributes to protein kinase $\mathrm{C}$ potentiation of vanilloid receptor activity. J Biol. Chem. 2004, 279, 25665-25672.

28. Smith, C.P.; Vemulakonda, V.M.; Kiss, S.; Boone, T.B.; Somogyi, G.T. Enhanced ATP release from rat bladder urothelium during chronic bladder inflammation: Effect of botulinum toxin A. Neurochem. Int. 2005, 47, 291-297.

29. Vemulakonda, V.M.; Somogyi, G.T.; Kiss, S.; Salas, N.A.; Boone, T.B.; Smith, C.P. Inhibitory effect on intravesically applied botulinum toxin A in chronic bladder inflammation. J. Urol. 2005, 173, 621-624. 
30. Guo, B.L.;Zheng, C.X.; Sui, B.D.; Li, Y.Q.; Wang, Y.Y.; Yang, Y.L. A closer look to botulinum neurotoxin type A-induced analgesia. Toxicon 2013, 71, 134-139.

31. Kuo, H.C.; Jiang, Y.H.; Tsai, Y.C.; Kuo, Y.C. Intravesical botulinum toxin-A injections reduce bladder pain of interstitial cystitis/bladder pain syndrome refractory to conventional treatmentA prospective, multicenter, randomized, double-blind, placebo-controlled clinical trial. Neurourol. Urodyn. 2015, doi:10.1002/nau.22760.

32. Homma, Y.; Ueda, T.; Tomoe, H.; Lin, A.T.; Kuo, H.C.; Lee, M.H.; Lee, J.G.; Kim, D.Y.; Lee, K.S. Clinical guidelines for interstitial cystitis and hypersensitive bladder syndrome. Int. J. Urol. 2009, 16, 597-615.

33. Lubeck, D.P.; Whitmore, K.; Sant, G.R.; Alvarez-Horine, S.; Lai, C. Psychometric validation of the O'Leary-Sant interstitial cystitis symptom index in a clinical trial of pentosan polysulfate sodium. Urology 2001, 57, 62-66.

34. Propert, K.J.; Mayer, R.D.; Wang, Y.; Sant, G.R.; Hanno, P.M.; Peters, K.M.; Kusek, J.W. Responsiveness of symptom scales for interstitial cystitis. Urology 2006, 67, 55-59.

35. Abrams, P.; Cardozo, L.; Fall, M.; Griffiths, D.; Rosier, P.; Ulmsten, U.; van Kerrebroeck, P.; Victor, A.; Wein, A. The standardisation of terminology of lower urinary tract function: Report from the standardisation sub-committee of the international continence society. Neurourol. Urodyn. 2002, 21, 167-178.

(C) 2015 by the authors; licensee MDPI, Basel, Switzerland. This article is an open access article distributed under the terms and conditions of the Creative Commons Attribution license (http://creativecommons.org/licenses/by/4.0/). 